\title{
Apoptosis Detection for Adherent Cell Populations in Time-Lapse Phase-Contrast Microscopy Images
}

\author{
Seungil Huh ${ }^{1}$, Dai Fei Elmer Ker ${ }^{2}$, Hang $\mathrm{Su}^{1}$, and Takeo Kanade ${ }^{1}$ \\ 1 Robotics Institute, Carnegie Mellon University \\ \{seungilh, hangs, tk\}@cs.cmu.edu \\ 2 Department of Orthopedic Surgery, Stanford University \\ elmerker@stanford.edu
}

\begin{abstract}
The detection of apoptosis, or programmed cell death, is important to understand the underlying mechanism of cell development. At present, apoptosis detection resorts to fluorescence or colorimetric assays, which may affect cell behavior and thus not allow long-term monitoring of intact cells. In this work, we present an image analysis method to detect apoptosis in time-lapse phase-contrast microscopy, which is nondestructive imaging. The method first detects candidates for apoptotic cells based on the optical principle of phase-contrast microscopy in connection with the properties of apoptotic cells. The temporal behavior of each candidate is then examined in its neighboring frames in order to determine if the candidate is indeed an apoptotic cell. When applied to three $\mathrm{C} 2 \mathrm{C} 12$ myoblastic stem cell populations, which contain more than 1000 apoptosis, the method achieved around $90 \%$ accuracy in terms of average precision and recall.
\end{abstract}

Keywords: Apoptosis detection, Time-lapse phase-contrast microscopy, Microscopy image restoration, Event detection in videos.

\section{Introduction}

The detection of apoptosis, or programmed cell death, is critical for furthering our understanding in biology as apoptosis plays a significant role in both normal tissue development and disease progression, e.g., proper organ development, stress-induced neurodegeneration, and cancer cell development. In addition, apoptosis detection is often used for toxicity screening of compounds, such as pharmacological reagents and biomaterials, as well as drug discovery and subsequent dosage optimization of chemotherapeutic agents.

Apoptosis occurs in an orderly, step-wise manner starting with a series of biochemical events that lead to characteristic changes in the cell prior to its death. The process of apoptosis includes cell shrinking, membrane blebbing, DNA degradation, and the formation of apoptotic bodies that serve to minimize spillage of the internal contents of a dying cell to its surroundings [1].

N. Ayache et al. (Eds.): MICCAI 2012, Part I, LNCS 7510, pp. 331-339, 2012.

(C) Springer-Verlag Berlin Heidelberg 2012 
Presently, apoptosis is detected using a variety of assays, which include absorbance measurements and fluorescence or colorimetric stains, to measure the levels and activity of apoptotic molecules. These procedures often require a sample to be harvested for each time-point measurement; thus, long-term cell monitoring is not feasible and multiple samples may be required. On the other hand, image analysis of cells using non-destructive imaging such as phase-contrast microscopy offers a way to monitor and detect apoptosis in a population of cells over time without adversely affecting cell behavior or requiring additional samples.

In this work, we present a method to detect apoptosis in time-lapse phasecontrast microscopy, particularly for adherent cells, which involves changes in cell morphology and image intensity during apoptosis. The method first detects the cells that shrink and become bright as candidates for apoptotic cells in order to reduce the search space. For this candidate detection, we propose a computational model of phase-contrast microscopy that can be used to detect both bright and dark cells. Each candidate is examined to determine if apoptosis indeed occurs based on changes in image intensity and texture over the neighboring frames. The proposed method was tested on three time-lapse microscopy image sequences of $\mathrm{C} 2 \mathrm{C} 12$ myoblastic stem cells.

\section{$1.1 \quad$ Related Work}

There have been little-to-no reports of apoptosis detection in phase-contrast microscopy. To the best of our knowledge, cell death event detection has only been implicitly performed as a byproduct of cell tracking; i.e., if the trajectory of a cell terminates during cell tracking, the cell is considered dead. However, this simple heuristic often yields poor results because many cell trajectories terminate due to failures in cell tracking as opposed to actual cell death.

One may think that apoptosis detection can be performed by the methods for mitosis (cell division) detection, such as the method in [2]. However, such methods are not effective because mitosis detection depends on a unique visual presentation lasting only a short time, namely a figure eight shape, while apoptosis does not involve such a distinctive visual hallmark. In addition, after cells die through apoptosis, the dead cells often form a cluster with other living or dead cells, which makes apoptosis detection more difficult than mitosis detection.

\section{Method}

To narrow down the locations where apoptosis begins, we first locate bright cells whose formation is followed by size shrinkage and brightness increase. Each candidate is then validated based on temporal changes in brightness and texture.

\subsection{Cell Region Detection}

To detect apoptosis candidates, we detect both bright and dark cell areas using a computational model for the optical principle of phase-contrast microscopy.

According to 3], phase-contrast imaging can be modeled by two waves: the unaltered surround wave $\tilde{l}_{S}(x)$ and the diffracted wave $\tilde{l}_{D}(x)$, computed as 


$$
\begin{aligned}
& \tilde{l}_{S}(x)=i \zeta_{p} A e^{i \beta} \\
& \tilde{l}_{D}(x)=\zeta_{c} A e^{i(\beta+f(x))}+\left(i \zeta_{p}-1\right) \zeta_{c} A e^{i(\beta+f(x))} * \operatorname{airy}(r)
\end{aligned}
$$

where $i^{2}=-1 ; A$ and $\beta$ are the illuminating wave's amplitude and phase before hitting the specimen plate, respectively; $\zeta_{p}$ and $\zeta_{c}$ are the amplitude attenuation factors by the phase ring and the specimen, respectively; $f(x)$ is the phase shift caused by the specimen at location $x$; and, airy $(r)$ is an obscured Airy pattern.

The intensity of the final observed image $g(x)$ is then computed as

$$
\begin{aligned}
g(x) & =\left|\tilde{l}_{S}(x)+\tilde{l}_{D}(x)\right|^{2} \\
& =\left|i \zeta_{p} A e^{i \beta}+\zeta_{c} A e^{i(\beta+f(x))}+\left(i \zeta_{p}-1\right) \zeta_{c} A e^{i(\beta+f(x))} * \operatorname{airy}(r)\right|^{2} \\
& \approx 2 \zeta_{c} \zeta_{p}\left(1+\zeta_{c}\right) A^{2}\left(\frac{\zeta_{p}\left(1+\zeta_{c}\right)}{2 \zeta_{c}}+f(x)-f(x) * \operatorname{airy}(r)\right) \\
& \propto f(x) *(\delta(r)-\operatorname{airy}(r))+C
\end{aligned}
$$

where $C=\frac{\zeta_{p}\left(1+\zeta_{c}\right)}{2 \zeta_{c}}$ is a constant. Based on this approximate linear relation between $f(x)$ and $g(x)$ in Eq. (6), $f(x)$ can be reconstructed from $g(x)$. Since $f(x)$ is the phase shift caused by the specimen, thresholding $f(x)$ results in the detection of cell areas [3].

Note that in order to obtain Eq. (5) from Eq. (4), it is assumed that $f(x)$ is close to zero, based on which following three approximations are applied:

$$
e^{i f(x)} \approx 1+i f(x), \quad f(x)^{2} \approx 0, \quad(f(x) * \operatorname{airy}(r))^{2} \approx 0 .
$$

However, these approximations are not valid particularly for bright cells, which cause greater phase retardations than dark cells 1 . As a result, the method has difficulty in detecting mitotic or apoptotic cells, which appear bright due to their increased thickness.

To detect bright cells (and also dark cells more properly), we generalize the model by assuming that $f(x)$ is close to a certain phase $\theta$, which is not necessarily zero. More formally, $f(x)$ is replaced with $\theta+\tilde{f}(x)$, where $\theta$ is a constant and $\tilde{f}(x)$ is close to zero. Based on this relaxed assumption, $g(x)$ is computed as

$$
g(x)=\left|i \zeta_{p} A e^{i \beta}+\zeta_{c} A e^{i(\beta+\theta+\tilde{f}(x))}+\left(i \zeta_{p}-1\right) \zeta_{c} A e^{i(\beta+\theta+\tilde{f}(x))} * \operatorname{airy}(r)\right|^{2}
$$

to which we apply the following approximations

$$
e^{i \tilde{f}(x)} \approx 1+i \tilde{f}(x), \quad \tilde{f}(x)^{2} \approx 0, \quad(\tilde{f}(x) * \operatorname{airy}(r))^{2} \approx 0,
$$

\footnotetext{
${ }^{1}$ Even for the detection of dark cells, the assumption $f(x) \approx 0$ is not quite valid because the diffracted wave is retarded in phase by approximately 90 degrees through interaction with the specimen 4 .
} 

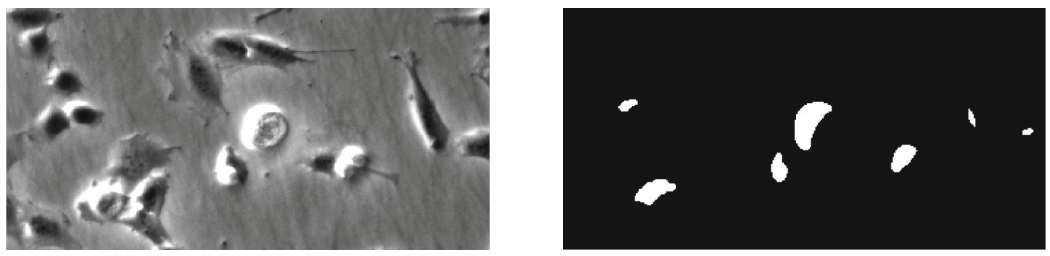

Fig. 1. A sample image and bright cell areas on it detected by the proposed model

(a)
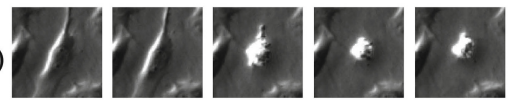

(b)
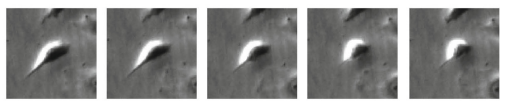

Fig. 2. Apoptosis processes in consecutive frames. An apoptotic cell shrinks and its brightness increases (a) abruptly for a short time period (less than five minutes) or (b) gradually for a long time period (tens of minutes or a few hours).

resulting in

$$
\begin{aligned}
& g(x) \approx 2 \zeta_{c} \zeta_{p}\left(\cos \theta+\zeta_{c}\right) A^{2} \\
& \quad \times\left(\frac{\zeta_{p}\left(1+\zeta_{c}^{2}+2 \zeta_{c} \cos \theta\right)}{2 \zeta_{c}\left(\cos \theta+\zeta_{c}\right)}+\tilde{f}(x)-\frac{\cos \theta+\zeta_{c}+\zeta_{p} \sin \theta}{\cos \theta+\zeta_{c}} \tilde{f}(x) * \operatorname{airy}(r)\right) \\
& \propto \tilde{f}(x) *(\delta(r)-B \cdot \operatorname{airy}(r))+C^{\prime}
\end{aligned}
$$

where $B=\frac{\cos \theta+\zeta_{c}+\zeta_{p} \sin \theta}{\cos \theta+\zeta_{c}}$ and $C^{\prime}=\frac{\zeta_{p}\left(1+\zeta_{c}^{2}+2 \zeta_{c} \cos \theta\right)}{2 \zeta_{c}\left(\cos \theta+\zeta_{c}\right)}$. Note that, if $\theta=0$, then $\tilde{f}(x)=f(x), B=1$, and $C^{\prime}=C$; thus, Eq (11) reduces to Eq (6). Since this is also a linear relation between $\tilde{f}(x)$ and $g(x), \tilde{f}(x)$ can be reconstructed from $g(x)$ and cell areas can be detected by thresholding $\tilde{f}(x)$.

Using this model, we detect bright and dark cell areas, separately with two different parameters: $\theta_{b}$ and $\theta_{d}$. For the parameter setting, we tested several values $(0, \pi / 6, \cdots, 11 \pi / 6)$ and selected the best ones based on apoptosis detection accuracy on the training set. (This can also be conducted by visual examination on a first few images.) The proposed model can detect bright cells as well as dark cells, unlike the previous model. Fig. 1 shows bright cell areas detected by our model, where bright halos are undetected or weakly detected.

\subsection{Apoptosis Candidate Detection}

To detect the cells that undergo the beginning of an apoptotic process, we examine each bright cell area to determine if its formation is followed by the decrease of dark area and/or the increase of bright area, which represent cell shrinkage and brightness increase, respectively. If the change is not trivial, the bright cell area is considered a candidate for an apoptotic cell; otherwise, it is regarded an already dead cell or a bright halo, and thus is not further taken into account. 


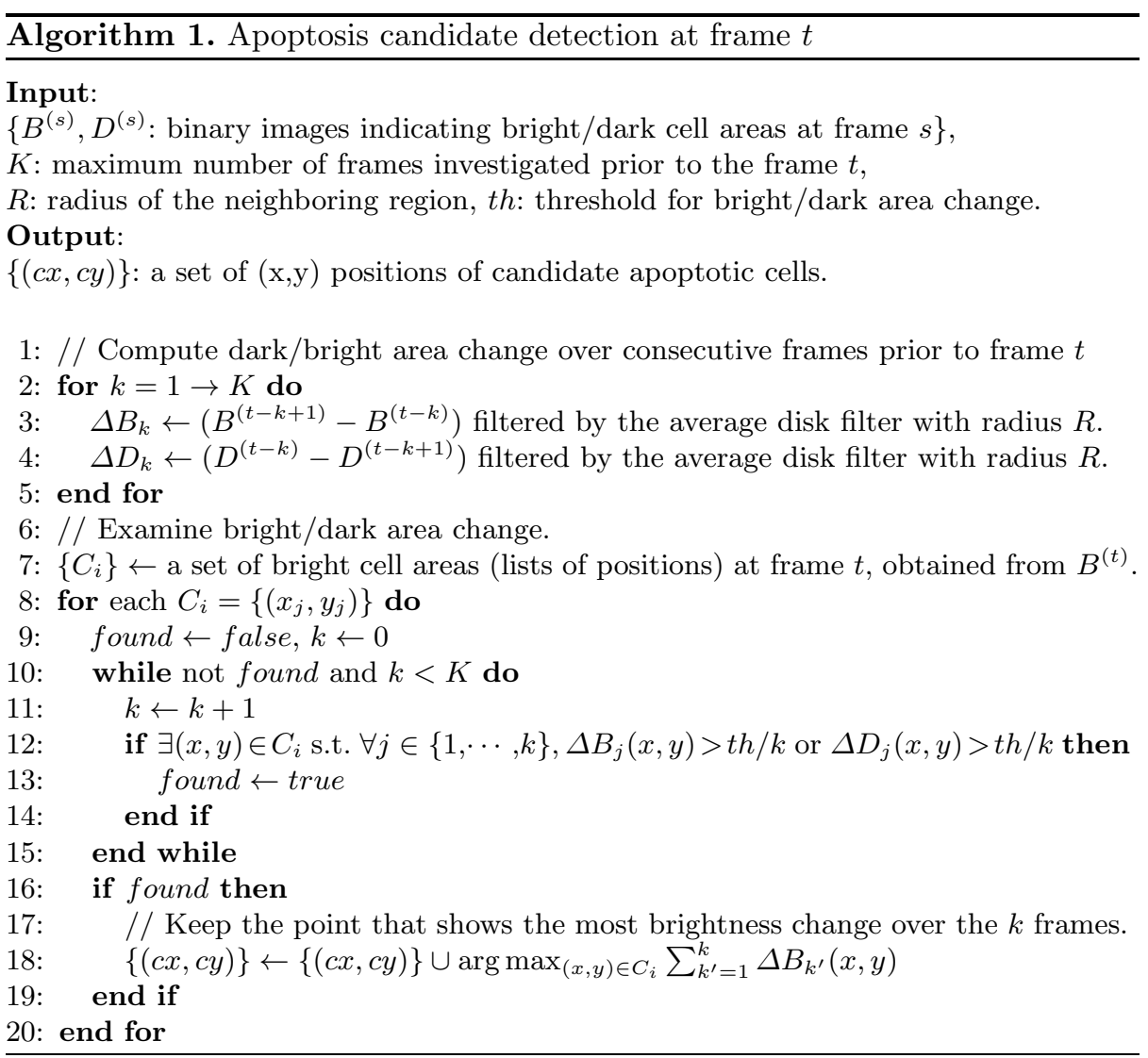

More formally, for each bright cell area at frame $t$, we examine its neighboring region over consecutive frames prior to the frame $t$. If the proportion of the bright area expanded or dark area shrunk in the region to the region's area is greater than a certain threshold, then the bright cell area is considered a candidate for an apoptotic cell. As the duration of cell shrinking and brightness change varies (See Fig. 2, ) and the image acquisition interval can also be different among experiments, we investigate different numbers of frames (up to $K$ frames) prior to frame $t$. More specifically, if the change between every two consecutive frames among the $k+1$ preceding frames is greater than the reduced threshold $t h / k$ for any $k \in\{1, \cdots, K\}$, then the bright cell area is considered a candidate for an apoptotic cell. The detailed procedure of apoptosis candidate detection is described in Algorithm 1 .

The neighboring region is set to be a circle with radius $R$. Hence, this scheme involves three parameters: $K, R$, and $t h$. We set these parameters to achieve at least a certain high level of recall (e.g., 99\%) and as high a precision as possible in candidate detection among the training data (Note that as th decreases, recall increases while precision decreases.). $K$ and $R$ can also be determined based on 
(a)

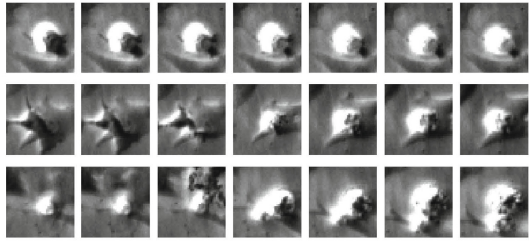

(d)

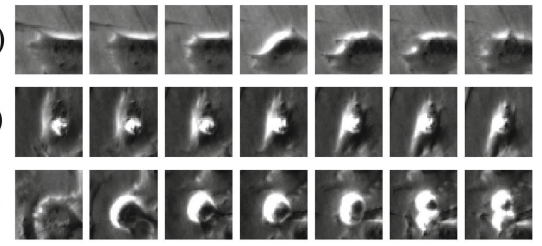

Fig. 3. Candidate patch sequences containing apoptosis (left) and non-apoptosis (right): (a-c) apoptotic cells in contact with none, a living cell, and a (or a group of) dead cell(s); (d-f) a change of halo, a (or a group of) dead cell(s), and mitosis

the observation of apoptosis duration and cell size/movement, respectively. In our experiments, $K, R$, and th were set to be 5,10 , and 0.25 , respectively.

\subsection{Feature Extraction}

Each candidate is tracked in the neighboring frames in order to incorporate temporal information by using a standard correlation tracking method, resulting in candidate patch sequences as shown in Fig. 3. Investigating temporal information helps to avoid detecting mitosis, which shows similar visual change to apoptosis at the beginning, as well as bright halos and dead cells. This step involves two parameters, the size of patch and the number of frames tracked on one side (preceding or following a candidate), which can be set by a typical validation scheme. In our experiments, they were set to be 50 pixels and 3 patches. From each patch in a patch sequence, we extract the following features:

- Brightness change histogram binning to 16 bins,

- Rotation invariant uniform local binary pattern (LBP ${ }^{\text {riu2 }}$ ) [5].

The former, which is computed on the difference between a patch and its previous patch, captures brightness change over time, the major cue for apoptosis detection, more precisely and in more detail than the candidate detection step. The latter captures the texture property of apoptotic cells, which is quite different from that of non-apoptotic cells as apoptosis involves membrane blebbing and the formation of apoptotic bodies. It is worth mentioning that these features are robust to global illumination change due to experimental setting.

\subsection{Candidate Validation}

We applied a linear Support Vector Machine (SVM) to classify candidate patch sequences. We tested several other classifiers used for mitosis detection, Hidden Conditional Random Field (HCRF) 6] and its variations [2 7]. All these classifiers as well as an RBF kernel SVM did not outperform a linear SVM despite their higher computational cost, presumably because visual features of apoptosis are less informative and more noisy in the sense that apoptosis does not 

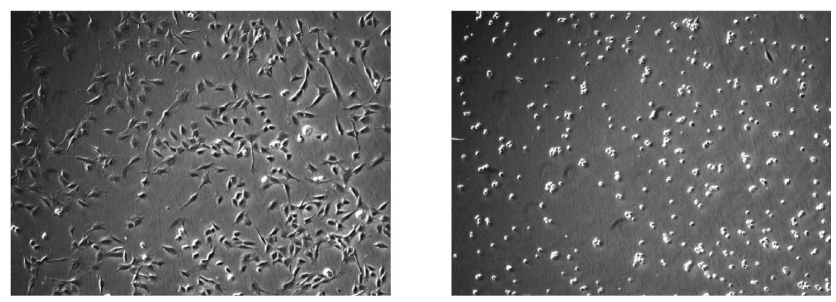

Fig. 4. The first (left) and last (right) frames of the data

involve distinctive morphological features, such as a figure eight shape during mitosis. Under such a circumstance, a max-margin classifier with a simple decision boundary might be more effective to eliminate outliers or meaningless patterns. After classification, the post-processing in [7] is conducted to prevent one apoptosis from being detected multiple times.

\section{$3 \quad$ Experiments}

We introduce the experimental setup and results with discussions.

\subsection{Image and Ground Truth Acquisition}

After C2C12 myoblastic stem cells were cultured for one day, Mitomycin C was added to induce apoptosis. Afterward, three populations were imaged every 5 minutes over 45 hours, resulting in three sets of 540 image frames. As shown in Fig. 4. most of cells were dead at the last frame. We manually annotated apoptosis by marking the center of each apoptotic cell after it shrinks and becomes bright, obtaining 1154 cases in total. The image sequences and ground truths are available on the first author's web page (www.cs.cmu.edu/ seungilh).

\subsection{Evaluation}

A detection is considered a true positive if an apoptotic cell is detected within spatially 30 pixels and temporally 3 frames from an annotated location. If an apoptotic cell is detected more than once, the only one that temporally the closest to the ground truth was considered true positive, the others false positives.

We used one sequence as a training set and another one as a test set, testing all six training-testing set pairs. We set all the parameters including the SVM parameter through a four-fold cross validation on the training set.

\subsection{Results and Discussions}

Our method achieved an average precision of $93.0 \% \pm 1.1 \%$ and an average recall of $89.8 \% \pm 1.4 \%$ for apoptosis detection. False positives mostly happened due to 
(a)

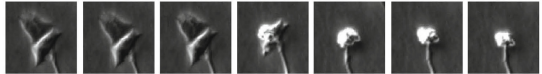

(b)

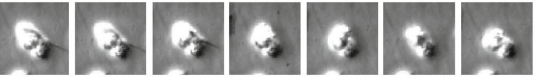

Fig. 5. Examples of undetected apoptosis after candidate validation. (a) As two cells in contact with each other undergo apoptosis simultaneously, only one apoptosis is detected. (b) An apoptotic cell is barely observable as it is covered with two dead cells.

(a)

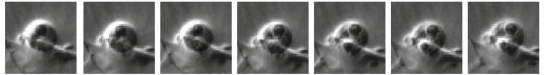

(b)

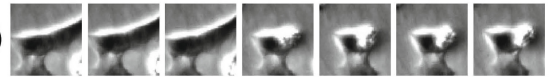

Fig. 6. Examples of undetected apoptosis at the candidate detection step. (a) Apoptosis occurs without brightness change. (b) As a small apoptotic cell is located in contact with a compact and dark cell, the bright apoptotic cell is considered the bright halo of the dark cell and thus not detected at the bright cell area detection step.

rapidly changing halos and moving dead cells attached to living cells. Duplicate detection sometimes occurred as apoptotic cells abruptly and considerably move while they shrink or when cell internal contents spill out. False negatives mostly happened when cells form a cluster in which multiple apoptosis simultaneously occur or apoptotics cells are occluded by other cells, as shown in Fig. 5 .

In the candidate detection step, our method detected almost all apoptosis except a few cases (See Fig. 6]). The number of candidates were approximately three times as many as the number of apoptosis.

Our model-based cell area detection is effective, particularly when cell density is high and thus cells are in contact with one another and halos appear among them. The scheme outperformed a cell area detection scheme based on intensity thresholding by $3.3 \%$ and $10.3 \%$ in terms of average apoptosis detection precision and recall, respectively, on the last 100 frames of the three sequences.

\section{Conclusion}

We have presented an apoptosis detection method for adherent cells that detects apoptosis candidates and then validates them. For the candidate detection, we proposed a cell area detection method based on the optical principle of phase-contrast microscopy. When applied to three cell populations, our method achieved around $90 \%$ accuracy in terms of average precision and recall.

\section{References}

1. Fuchs, Y., Steller, H.: Programmed cell death in animal development and disease. Cell 147(4), 742-758 (2011)

2. Huh, S., et al.: Automated mitosis detection of stem cell populations in phasecontrast microscopy images. IEEE Trans. Med. Imaging 30(3), 586-596 (2011) 
3. Yin, Z., Kanade, T., Chen, M.: Understanding the phase contrast optics to restore artifact-free microscopy images for segmentation. Med. Image Anal. 16(5), 1047-1062 (2012)

4. http://www.microscopyu.com/articles/phasecontrast/phasemicroscopy.html

5. Ojala, T., Pietikäinen, M., Mäenpää, T.T.: Multiresolution gray-scale and rotation invariant texture classification with local binary pattern. IEEE Trans. Pattern. Anal. Mach. Intell. 24(7), 971-987 (2002)

6. Quattoni, A., Wang, S., Morency, L., Collins, M., Darrell, T.: Hidden conditional random fields. IEEE Trans. Pattern Anal. Mach. Intell. 29(10), 1848-1853 (2007)

7. Huh, S., Chen, M.: Detection of mitosis within a stem cell population of high cell confluence in phase-contrast microscopy images. In: IEEE CVPR, pp. 1033-1040 (2011) 